\title{
Identical Approximation Operator and Regularization Method for the Cauchy problem of 2D Heat Conduction Equation
}

\author{
Shangqin $\mathrm{He}^{1}$ and Xiufang Feng ${ }^{1}$ \\ ${ }^{1}$ Ningxia University
}

December 9, 2020

\begin{abstract}
In this paper, an identical approximate regularization method is extended to the Cauchy problem of two-dimensional heat conduction equation, this kind of problem is severely ill-posed. The convergence rates are obtained under a priori regularization parameter choice rule. Numerical results are presented for two examples with smooth and continuous but not smooth boundaries, and compared the identical approximate regularization solutions which are displayed in paper. The numerical results show that our method is effective, accurate and stable to solve the ill-posed Cauchy problems.
\end{abstract}

\section{Hosted file}

heatequation2D.pdf available at https://authorea.com/users/382069/articles/498015-identicalapproximation-operator-and-regularization-method-for-the-cauchy-problem-of-2d-heatconduction-equation 